\title{
s-Channel Heliticity Conservation, Fixed Poles and Dip Mechanisms.
}

\author{
H. F. Joxes \\ Physics Department, Imperial College - London. \\ (Lett. Fuow Cimento, 4, 545 (1970))
}

The second paragraph on p. 548 regarding the dip nechanisms of $P^{\prime}$ and 1 is wrong, and indeed in confliet with Table I. It should be ancended to read:

"For the $P^{\prime}$ or (") s-channel helicity conservation implies that the coupling $g_{1}$ valuishes, which is incompatible with choosing sense or the che w mechanism ".

\section{$* * *$}

The author is grateful to Dr. Cr. II. Hite for pointing out this error. 